\title{
Codepoiesis - the Deep Logic of Life
}

\author{
Marcello Barbieri
}

Received: 10 May 2012 / Accepted: 27 July 2012 / Published online: 16 August 2012

(C) Springer Science+Business Media B.V. 2012

Modern Biology describes the cell as an 'autopoietic' system, a "system that fabricates itself'. The concept of autopoiesis, or self-production, describes the ability of living systems to produce their own components and eventually to produce copies of themselves. At a closer look, however, we realize that this is not always what takes place in real life. In embryonic development, for example, the cells produce cells that are different from their progenitors. They are also capable of something that is the very opposite of autopoiesis: they can suicide themselves.

It may be pointed out that embryonic cells appeared very late in evolution and derived from previous autopoietic systems, so it is autopoiesis that we must start with because it was at the beginning of everything. But this is precisely what we cannot say. Before the origin of the genetic code, specific proteins could not exist, and the ancestral systems were producing descendants that were inevitably different from themselves. They were not autopoietic systems. Autopoiesis, in other words, did not exist before the first cells, so it was not the mechanism that gave origin to them.

There is no trace of the first cells in the fossil record, but the existence of a nearly universal genetic code in all modern cells means that this code appeared in the progenitors of all living creatures, in systems that are collectively known as the common ancestor. We know very little of those ancestral systems, but the molecular data tell us that they had various types of RNA some of which were capable of joining peptides together into polypeptides.

Before the origin of the genetic code, that ancestral ribonucleoprotein system was engaged in the process of evolving coding rules and was therefore a code generating system. After the origin of the genetic code, however, the situation changed dramatically. No other modification in coding rules was accepted and the system in question became a code conservation system. Another part of the system, however, maintained the potential to evolve other coding rules and behaved as a new code generating, or code exploring, system. In the early Eukarya, for example, the cells had a code

M. Barbieri $(\bowtie)$

Dipartimento di Morfologia ed Embriologia, Via Fossato di Mortara 64a, 44121 Ferrara, Italy

e-mail: brr@unife.it 
conservation part for the genetic code, but also a code exploring part for the splicing code.

This tells us something important about life. The origin of the first cells was based on the ability of the ancestral systems to generate the rules of the genetic code, and the subsequent evolution of the cells was based on two complementary processes: one was the generation of new organic codes and the other was the conservation of the existing ones. Taken together, these two processes are the two sides of a biological phenomenon that can be referred to as "codepoiesis".

The ancestral systems that gave origin to the first cells, in conclusion, were not autopoietic systems but they had to be codepoietic systems. And all the cells that came after them in evolution were not always engaged in autopoiesis but were inevitably engaged in codepoiesis. What is always and necessarily present in all living systems, in other words, is codepoiesis, not autopoiesis.

\section{A New Definition of the Cell}

The data from molecular biology have revealed that all known cells belong to three primary kingdoms, or domains, that have been referred to as Archaea, Bacteria and Eucarya (Woese, 1987, 2000). The genetic code is the sole feature that the three domains have in common, and this suggests that the code appeared in primitive systems that had not yet developed the other main features of a modern cell design. In particular, they not yet acquired a signal transduction code. This code allows the cell to regulate protein synthesis according to the signals from the environment and was therefore extremely useful, which explains why there have been various independent attempts to develop it. It is a fact, at any rate, that Archaea, Bacteria and Eucarya have three distinct signalling systems, and this does suggest that each domain arose by the combination of the universal genetic code with three distinct signal-transduction codes.

Once in existence, the cells of the three primary kingdoms adopted strategies that channelled them into two very different evolutionary directions. Archaea and Bacteria chose a streamlining strategy that eliminated all redundant processes, including the potential to generate new organic codes, and have remained substantially the same ever since. This is dramatically illustrated by the discovery that modern stromatolites built by cyanobacteria are virtually identical to the 3.4 and the 1.8 billion year old stromatolites that have been found in the fossil record (Schopf 1999; Knoll 2003). The bacteria, in conclusion, have maintained the same fundamental structure for billions of years, and this makes of them the best known example of autopoietic systems.

The Eucarya, on the contrary, continued to explore the coding space and evolved new organic codes throughout the three thousand million years of cellular evolution. The splicing codes, for example, were instrumental to the origin of the nucleus, the histone code provided the rules of chromatin regulation, and the cytoskeleton codes allowed the eukarya to perform internal movements, including those of mitosis and meiosis (Barbieri 2003).

The bacteria, in conclusion, are true autopoietic systems, whereas the ancestors of the first cells and the eukarya are codepoietic systems. This shows that autopoiesis is a special case of codepoiesis. More precisely, autopoiesis takes place when a system perpetuates its own codes but has lost the ability to generate new ones. What is 
common to all living systems, therefore, is either the generation or the conservation, or both, of organic codes. This gives us an entirely new definition of the cell that can be expressed in this way: "the cell is a codepoietic system, i.e., a system that is capable of creating and conserving its own codes".

This definition accounts for the two most important events that took place in evolution. The ability to create coding rules accounts for the origin of the genetic code and of all the other codes that followed. The ability of the cell to conserve its own codes accounts for the fact that the organic codes are the great invariants of life, the entities that must be conserved while everything else is changing.

We realize in this way that living systems do not need to produce all of their components in order to stay alive: what is imperative is the perpetuation of their codes, whereas all other features can change within ample limits. The deep logic of life, in conclusion, is codepoiesis, not autopoiesis.

We conclude that codepoiesis, the generation and the conservation of organic codes, was the main driving force of evolution, and Code biology, the study of all codes of life, from the genetic code all the way up to the codes of language, is truly the new frontier of biology.

\section{References}

Barbieri, M. (2003). The organic codes. An introduction to semantic biology. Cambridge, UK: Cambridge University Press.

Knoll, A. H. (2003). Life on a young planet. The first three billion years of evolution on Earth. Princeton NJ: Princeton University Press.

Schopf, J. W. (1999). Cradle of life: The discovery of Earth's earliest fossils. Princeton NJ: Princeton University Press.

Woese, C. R. (1987). Bacterial evolution. Microbiol Reviews, 51, 221-271.

Woese, C. R. (2000). Interpreting the universal phylogenetic tree. Proceedings of the National Academy of Sciences USA, 97, 8392-8396. 